\title{
Self-assembly of fine particles along tool-mark produced by free-form cutting for improving biochemical analysis *
}

\author{
- Wettability modification with controlled cutting conditions -
}

Yuji OKUBO $^{* *}$ and Nobuyuki MORONUKI ${ }^{* * *}$

\begin{abstract}
Self-assembly of fine particles on three-dimensional structure enables a lot of applications. Dip-coating is one of the processes to produce such assemblies and often utilized because of high productivity and simplicity, just drawing up from suspension in which particles are dispersed. However, it has been applied only to planar substrates because the spreading profile of suspension is constant when the drawing up speed is constant while that on three-dimensional substrates becomes complex. This study aims to realize uniform assembly on the three-dimensional structure with the texture fabricated using a five-axis milling machine. First, the spreading profile of water on directionally slanted grooves on planar substrate was investigated. It was found that water is well held by pinning and the effect becomes highest when the slant angle $30^{\circ}$. It was also found that the coverage increased when the groove pitch $100 \mu \mathrm{m}$ and the drawing up speed 12.5 $\mathrm{mm} / \mathrm{s}$. Then, assembly on the three-dimensional profile was tried. Dip-coating process was carried out referring the appropriate conditions for planar substrates. The effectiveness of the texture was verified comparing the assembly results on parabolic surface with/without it. Finally, it was found the uniform assembly on parabolic surface is applicable to optical element.
\end{abstract}

Key words: self-assembly, tool-mark, texture, wettability, particles

\section{Introduction}

Functional particles are utilized in various applications, such as biochemical analysis ${ }^{12)}$, dye-sensitized solar cell ${ }^{3}$, optical element $^{4)}$ and gas sensor ${ }^{5}$, in which particles should be placed at the required position. Among them, the surface of biochemical analysis systems is modified with proteins to react with specific chemical and emit fluorescent light by particles. With decrease in the particle size, specific surface area increases which enhance reaction at the surface according to the scaling law. Thus, finer particles are preffered ${ }^{6) 7}$. To improve the sensitivity of such biochemical analysis system further, combination of fine particle fluorescent with optical system should be considered as shown in Fig. 1. For example, if the particles located on a parabolic surface emit fluorescent light, the emission can be focus on the focal point to increase the intensity.

So far, self-assemblies of fine particles have been carried out only for planer substrates ${ }^{8}$. Typical one is dip-coating; dipping a hydrophilic substrate in aqueous suspension in which particles are dispersed, the suspension spread uniformly on the substrate. Then, the substrate is drawn up at constant speed and at fixed angle from the suspension. At the contact line or the edge of spreading, surface tension becomes dominant and meniscus force attracts neighboring particles. Finally, packed assembly of the particles are obtained as shown in Fig. 1. Physical model has been already established for planar substrate ${ }^{9)}$. However, self-assembly on a three-dimensional surface is not easy because the spreading of the suspension and recession speed of the contact line become different depending on the line position.

Textured surface has various kind of functions such as friction ${ }^{(0)-12)}$ and optical properties ${ }^{13)}$. Wettability of the surface is also affected by the texture ${ }^{14)}{ }^{15}$. Contact angle of a droplet can be changed by changing the texture size on the substrate. Various models have been proposed. Pinning is a big problem in the discussion of spreading of liquid on a surface. A liquid droplet is pinned just same with pinning a paper on a board at the boundary where physical and/or chemical property changes. Pinning changes not only the contact angle but the sliding angle, thus it

- Received 23 January 2015

Accepted 8 June 2015

Student member, Graduate School of System Design, Tokyo Metropolitan University (6-6, Asahigaoka, Hino-shi, Tokyo, 191-0065, JAPAN)

Full member, Faculty of System Design, Tokyo Metropolitan University (6-6, Asahigaoka, Hino-shi, Tokyo, 191-0065, JAPAN) can change the self-assembly process on a three-dimensional surface as shown in Fig. 2. It is expected that the particles selectively assemble along the texture pattern independent of the complex surface profile. However, there is no guideline to control the spreading of the suspension properly.

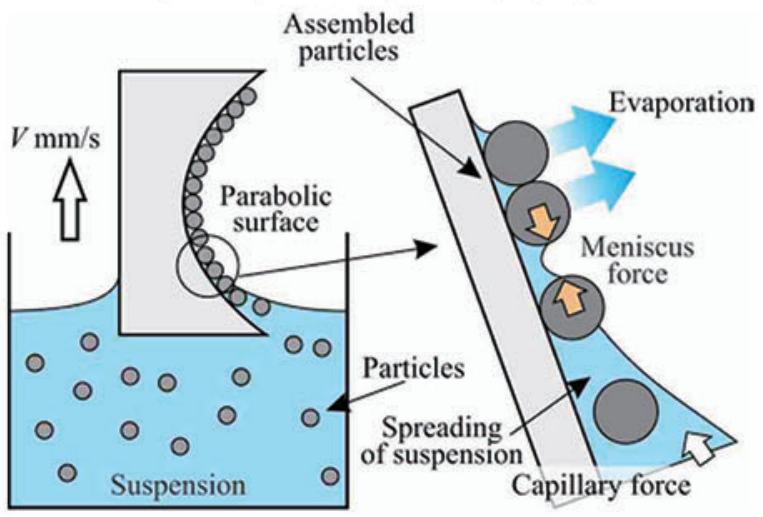

Fig. 1 Self-assembly of particles on free-form optical element and principle of self-assembly.

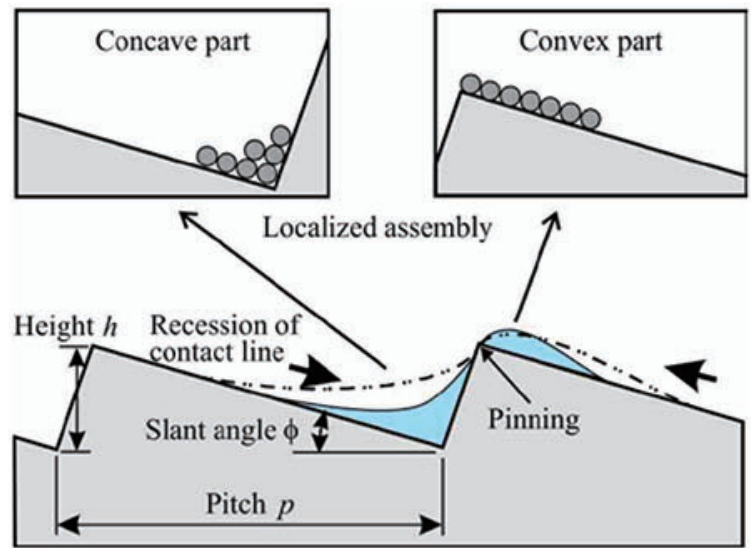

Fig. 2 Spreading of suspension on structured surface and localized self-assembly due to pinning of suspension. 
This paper aims to make clear the effect of fine grooves remained after cutting process not only on the spreading of the suspension but on the self-assembly process on it. The grooves are cut by controlling the feed and the tool inclination depending on the position utilizing CAM software as shown in Fig. 3.
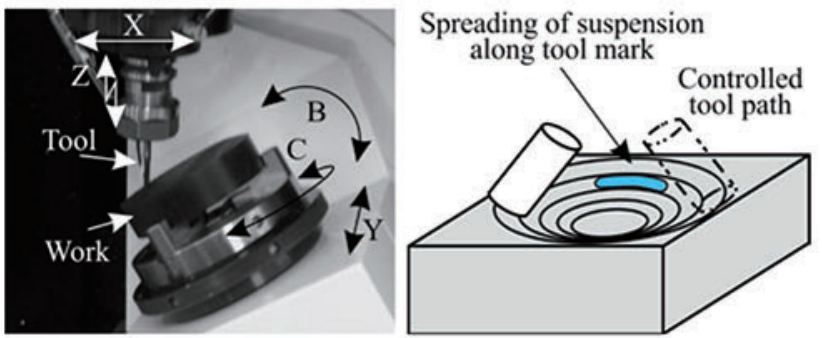

Fig. 3 Texturing of optical element utilizing tool-mark with controlled tool path.

\section{Effect of texture on spreading}

Before proceeding to the particle assembly process, preparation of the sample will be described and the spreading on the textured surface will be examined.

\subsection{Substrate fabrication}

Three dimensional profiles with fine grooves were machined by cutting with five-axes milling machine as already shown in Fig. 3. Typical conditions are summarized in Table 1. Tool path and its inclination data was produced with a CAM software (ESPRIT), while the profile was designed using a 3D CAD software (SolidWorks). The tool was $\phi 4 \mathrm{~mm}$ square end mill or ball mill, spindle speed was $10,000 \mathrm{~min}^{-1}$, and feed speed was set at $5000 \mathrm{~mm} / \mathrm{min}$. The workpiece material was a wax for modeling (ferris, melting point $120^{\circ} \mathrm{C}$ ) considering the easiness of machining. The grooves were made by cutting the workpiece while keeping the slant angle at constant. Then, the texture profile was replicated to another substrate made of polydimethylsiloxane (PDMS, Sylgard 184, curing condition $90^{\circ} \mathrm{C}, 2 \mathrm{~h}$ ) because the wettability of PDMS surface can be changed from hydrophobic to hydrophilic by exposing $\mathrm{O}_{2}$ plasma for $2 \mathrm{~min}$ (flow rate $20 \mathrm{sccm}, \mathrm{RF}$ Power $100 \mathrm{~W}$ ).

Table 1 Texturing conditions.

\begin{tabular}{c|c}
\hline \multicolumn{2}{c}{ Table 1 Texturing conditions. } \\
\hline Slant angle $\phi$ & $30,45,60^{\circ}$ \\
\hline Pitch $p$ & $0.2,0.4,0.8 \mathrm{~mm}$ \\
\hline Tool shape & Square / straight end mill $\phi 4 \mathrm{~mm}$ \\
\hline
\end{tabular}

\subsection{Spreading of water on the textured surface}

Texture profile in this study was as already defined in Fig. 2 The slant angle was set as a parameter to control the wettability while keeping the pitch and height at constant. The slant angle was set at 30 or $60^{\circ}$ toward the drawing up direction and pitch was typically $0.5 \mathrm{~mm}$. The substrate was set so that the grooves were parallel to water level. In this drawing up direction, water or suspension is pinned well and drag height is expected to be high. As a basic experiment, the substrate was draw up from pure water and the spreading was observed with a microscope as shown in Fig. 4.

From the figure the cross-sectional profile of the texture, zigzag in vertical direction, is observed. The liquid interface is also shown together with the sketch. The spreading profile is quite complex because of the pinning and the profile changes with the time elapsed. It was confirmed that the liquid is well held at the concave part due to the meniscus force. According to this complex spreading profile, the location of particle assembly discussed later should be specified. As shown in the figure, the slope where the water level climb up is called 'front-side', and another slope where the water level climb down is called 'back-side' later.

According to the previous study ${ }^{16) 17)}$, the film thickness after spreading and viscous drag can be calculated from capillary length $L \mathrm{c}$ (Eq. (1)) and capillary number $C$ a (Eq. (2)). The film thickness $t$ can be estimated from Eq. (3). The coverage $\varepsilon$ can be estimated from Eq. (4). Substituting typical number into these equations, it is derived that $V>10 \mathrm{~mm} / \mathrm{s}$ is appropriate condition when a planar substrate is drawn up from water ${ }^{18}$. . However, it is not easy to estimate these conditions for textured substrate due to the complicated effect of pinning and meniscus force on the wettability.

$$
\begin{aligned}
& L_{\mathrm{c}}=\sqrt{\frac{\gamma}{\rho \mathrm{g}}} \\
& C_{\mathrm{a}}=\frac{\mu V}{\gamma} \\
& t \approx L_{\mathrm{c}} \times \sqrt[3]{\mathrm{Ca}^{2}} \\
& \varepsilon=\frac{-\beta J_{e} \phi_{p}+V t\left(1-\phi_{p}\right)}{V t\left(1-\phi_{p}\right)}
\end{aligned}
$$

where $L \mathrm{c}$ : capillary length, $\gamma$. surface tension, $\rho$ : liquid density, $C$ a: capillary number, $\mu$ : viscosity, g: gravitational acceleration, $V$ : drawing up speed, $t$ : film thickness, $\beta$ : proportionality constant, $J_{e}$ : evaporation velocity, $\phi_{p}$ : volume fraction of particles.

In the discussion of the spreading of liquid, within an area smaller than the capillary length, surface tension becomes dominant and gravitational force can be neglected. In case of pure water, this length is about $2 \mathrm{~mm}$, thus, gravitational force can be neglected in this study.

Figure 5 shows the contact angle of the textured surface showing the effect of slant angle. The advancing contact angle is defined as the angle where liquid/vapor interface meet at a solid surface when the droplet expands while receding contact angle is the one when the droplet shrinks. Sliding angle is defined as the critical angle when the droplet begins to slide down on the inclined surface. The difference between advancing and receding contact angle is referred as contact angle hysteresis and well corresponds with the sliding angle. In the figure, the contact angle hysteresis is shown as a band between the plots of advancing-receding contact angles. It can be found from the figure that the hysteresis decreases with the increase in the slant angle almost independent of the pitch. Thus, smaller slant angle is considered as preferable to hold or keep much water on the texture.
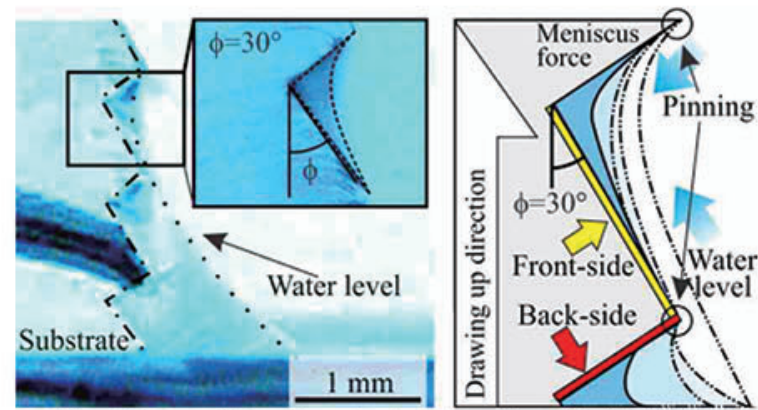

Fig. 4 Drag of liquid on textured surface $\left(\phi=30^{\circ}\right)$.

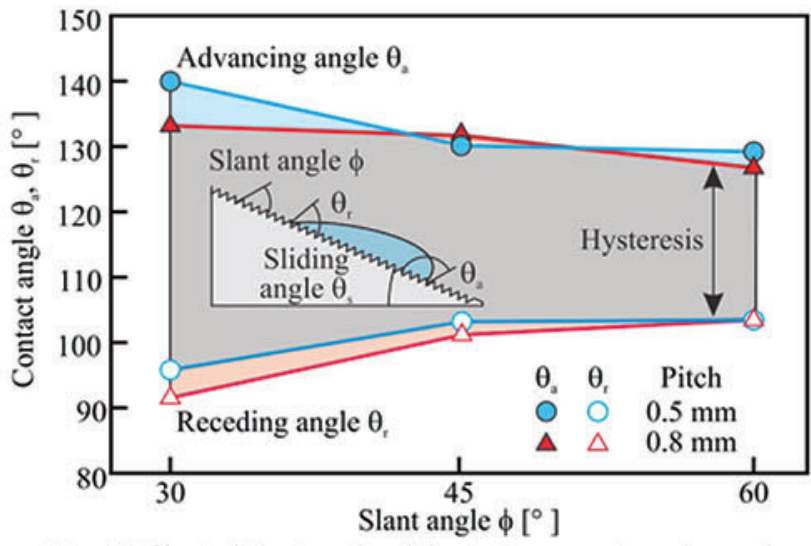

Fig. 5 Effect of slant angle of the texture on advancing and receding contact angle including the effect of texture pitch.

Figure 6 shows the results of drag height measurement. If the water is well hold on the surface, water is raise up together with the substrate which is drawn up at constant speed as estimated from Eq. (2). It is confirmed that smaller slant angle is preferable to hold or keep water on the surface. Also, as expected from the theory, faster speed $1 \mathrm{~mm} / \mathrm{s}$ is preferable. This speed is rather faster than the traditional dip coating process of several $\mu \mathrm{m} / \mathrm{s}$. 


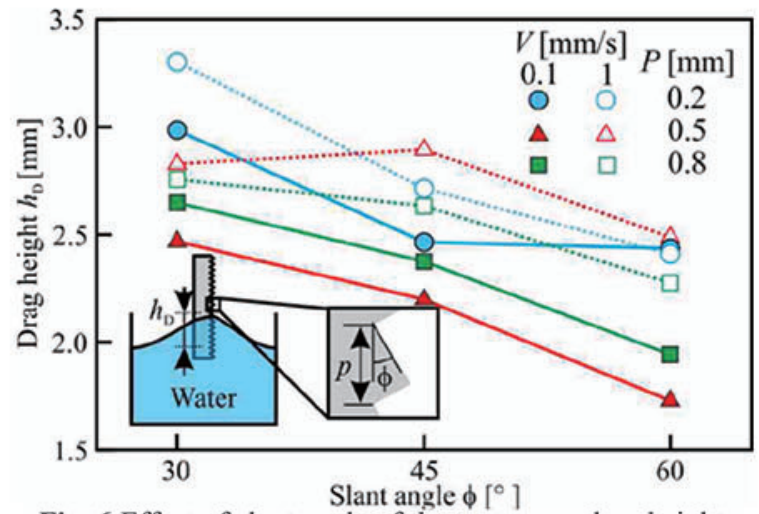

Fig. 6 Effect of slant angle of the texture on drag height.

To make clear that the effect of groove pitch of the texture on contact angle, dropping $10 \mathrm{~mL}$ of water, the contact angle was measured as shown Fig. 7. If the difference between contact angles between $30^{\circ}$ and $60^{\circ}$ of slant angle is too small, water cannot be trapped at concave part due to the effect of the pinning. When the pitch $p$ is $50 \mu \mathrm{m}$, the difference is little, while the pitch is wider than $100 \mu \mathrm{m}$, the difference becomes larger and thus the wider the pitch is, the larger the gap becomes. It is clear from the results that the pitch should be wider than $100 \mu \mathrm{m}$ to hold much water during the self-assembly. Based on these results, the texture pitch $100 \mu \mathrm{m}$ and slant angle $30^{\circ}$ is considered to be appropriate.

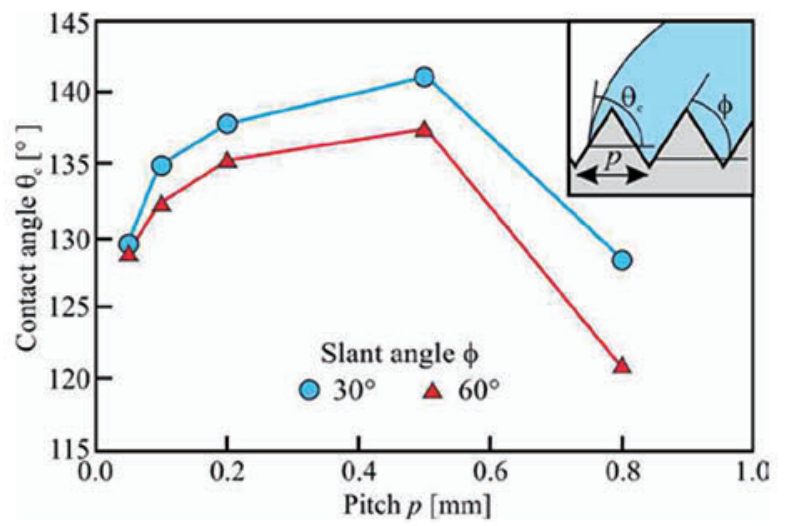

Fig. 7 Effect of groove pitch of the texture on contact angle.

\section{Effect of texture on self-assembly process}

To investigate the basic characteristic of particles assembly, texture was produced on a planar substrate and tested.

\subsection{Experimental procedure}

Silica particles, $1 \mu \mathrm{m}$ in diameter, were dispersed in water and assembled on the surface. Typical experimental conditions are summarized in Table 2. The surface tension of the suspension may be affected by the inclusion of particles and may change the capillary number. Thus, it was examined and compared with pure water with a surface tension balance. The surface tension of suspension was measured with Wilhelmy method as $72.50 \mathrm{mN} / \mathrm{m}$ while that of pure water was $72.75 \mathrm{mN} / \mathrm{m}$. The results are almost the same. So the spreading of suspension can be discussed just same with that of water. The interest of this study is the coverage of particles. The assembled particles were observed with a scanning electron microscope (SEM) and the coverage was calculated by analyzing the image with a free software "ImageJ" (NIH) after binarization. The maximum coverage of monolayer is about $80 \%$. Thus, the coverage over $80 \%$ means the structure is multilayered. Three samples were produced for each specification and the data were averaged. Regarding to the coverage, two different points were evaluated and averaged.

Table 2 Suspension and dip coating conditions.

\begin{tabular}{c|c|c|c}
\hline Suspension & Particle & Material & $\mathrm{SiO}_{2}$ \\
\cline { 3 - 3 } & & Diameter & $1 \mu \mathrm{m}$ \\
\cline { 2 - 3 } & & Concentration & $5 \mathrm{wt} . \%$ \\
\cline { 2 - 3 } & Solvent & Pure water \\
\hline \multirow{2}{*}{$\begin{array}{c}\text { Dip coating } \\
\text { condition }\end{array}$} & Drawing up speed & $0.1,1 \mathrm{~mm} / \mathrm{s}$ \\
\cline { 2 - 3 } & Drawing-up iteration & $1-5$ times \\
\hline
\end{tabular}

\subsection{Influence of drawing up speed on the coverage}

Figure 8 shows the effect of texture slant angle on the particle coverage on front-side. The error bar shows the maximum and minimum datum of three samples. Photos and cross-sectional schematic are shown upper part in the figure. It can be seen that the particles assembled along the grooves but the coverage is not necessary high. The part of 'front-side' is also shown in the schematic. It should be noticed that the surface area of the 'front-side' part differs depending on the slant angle. It is found from the lower graph that tendency of coverage became different depending on the drawing up speed. When the speed $V$ is low as $1 \mathrm{~mm} / \mathrm{s}$, the coverage increased with the slant angle, while it decreased when $V$ is high as $12.5 \mathrm{~mm} / \mathrm{s}$. This opposite tendency is reasonable considering the geometric symmetry. However, it is found from this result that high speed drawing is effective to obtain higher particle coverage. The reason is attributed to strong attraction of suspension due to the meniscus at the concave part of the texture.

Figure 9 shows the similar result of 'back-side'. The part 'back-side' is shown in the upper part of the figure. It is found from the lower graph that both of the slant angle and drawing up speed have little effect on the coverage comparing to the result on 'front-side' in Fig. 8. The suspension held at back-side is strongly attracted from front-side part of because the surface area is larger than that of 'back-side'. Thus, it is considered that the suspension is attracted to 'front-side' and the 'back-side' coverage becomes lower. Based on these results of Fig. 8 and 9 , it is concluded that slant angle $30^{\circ}$ and higher drawing up speed is required to obtain higher particle coverage.

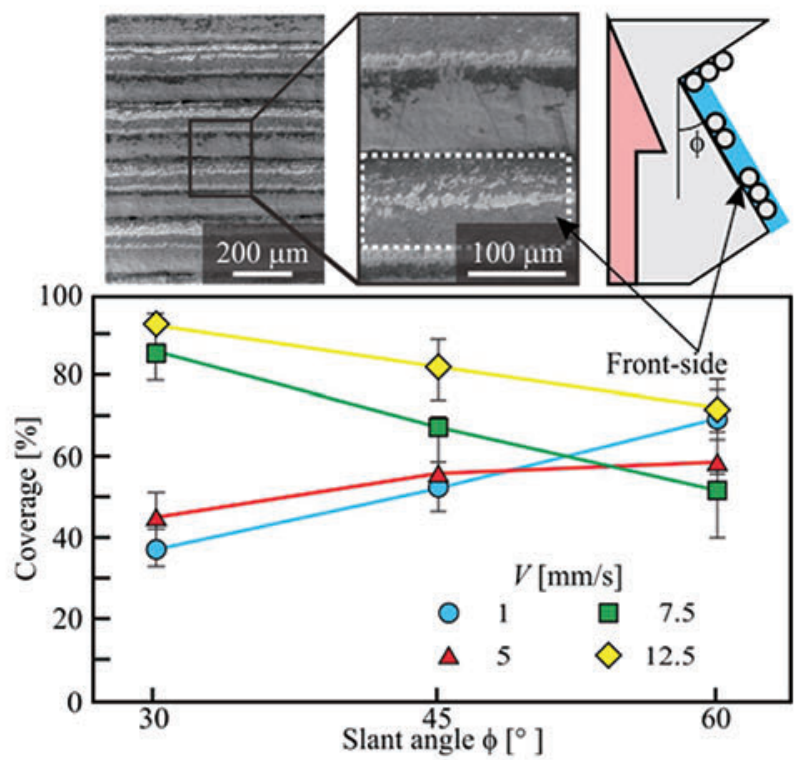

Fig. 8 Effect of the texture slant angle on front-side coverage.

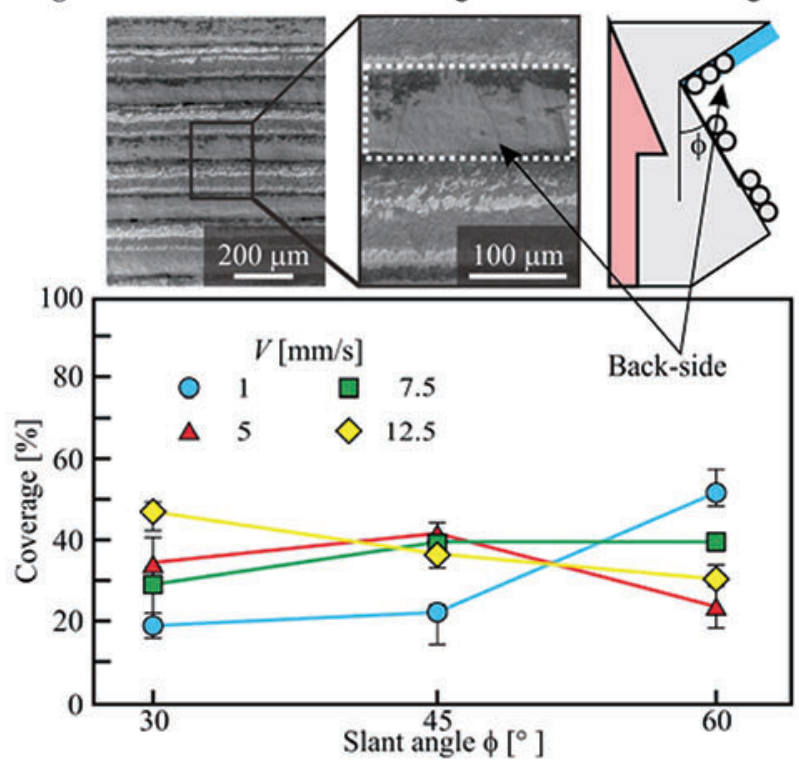

Fig. 9 Effect of the texture slant angle on back-side coverage. 


\subsection{Influence of texture pitch on coverage}

Based on the former results, the slant angle was fixed to $30^{\circ}$ and the effect of texture pitch was investigated as shown in Fig. 10. Drawing up speed was set at $10 \mathrm{~mm} / \mathrm{s}$ in this case. In the figure, the coverage is the summation of that on front-side and back-side. It is found that the coverage increases almost linearly with the decrease in pitch $P$. When the pitch is smaller than 200 $\mu \mathrm{m}$, the assembly covered all over the surface. As the texture pitch becomes larger, the assembly is difficult to be uniform and it is assembled to the specific site. Under these conditions, the assemblies looked multilayered though it was difficult to count the number of layers.

These results suggest that finer texture pitch $100 \mu \mathrm{m}$ can produce dense and uniform assembly. The reason is considered that increase in surface area thus higher evaporation rate may affect the assembly process. The important point is that the assembly site and alignment can be controlled with texturing.

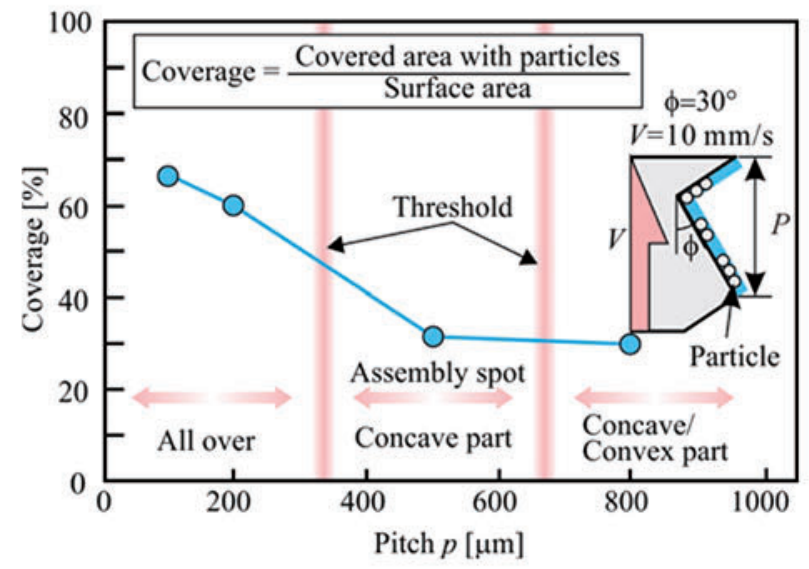

Fig. 10 Effect of the texture pitch on the coverage.

\subsection{Influence of drawing up times on coverage}

It is already known that the coverage increases with the number of iterations of dip coating process especially on planer and smooth substrate when the concentration of the particle is low. Figure 11 shows the effect of iteration number on the coverage. As this experiment, drawing up speed is $1 \mathrm{~mm} / \mathrm{s}$ because coverage is low on the former results. It is clear that the coverage increased with the increasing in the number. In the figure, the coverage on front-side can be compared with that in back-side. The coverage on back-side is always lower than that on front-side, but after five times iteration, the coverage reached $100 \%$. It is also confirmed that the coverage increases with the iterating numbers on textured surface as well as the case of smooth substrate.

It is interesting that the particles are not fixed on the texture during the iteration. In some cases on self-assembly on flat and smooth substrate, the assembly is dipped into strong acid to produce siloxane bond between silica particles and silicon substrate. This result suggests another aspect of fixation of particles, that is, textured surface can well hold the assembly, though the mechanism cannot be made clear here. The particles may stay on the surface only with Van der Waals force or the residual water molecules between the particle and the substrate.

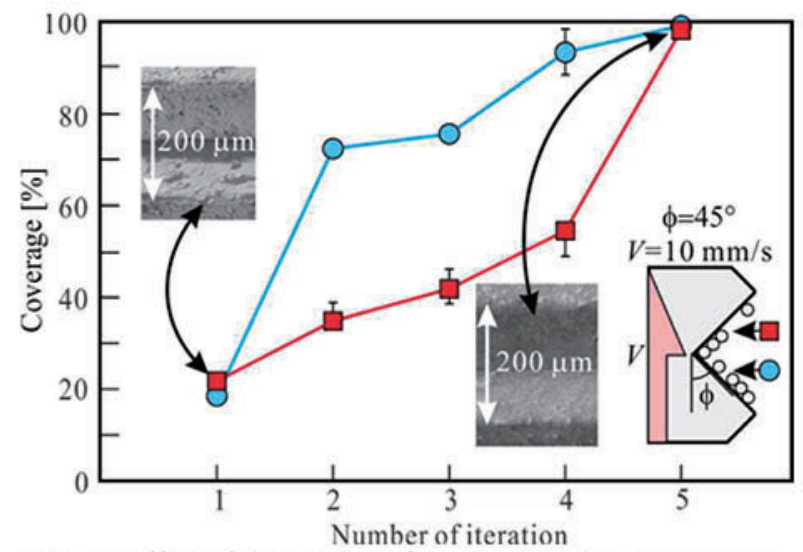

Fig. 11 Effect of the number of coating iteration on coverage.

\section{Particle assembly on parabolic surface}

In the final chapter, texture was produced on a three dimensional profile and particles were assembled on it. Moreover optical measurement was carried out.

4.1 Parabolic prifle preparation

A parabolic mirror gathers parallel incident light to a focal point. If fluorescent particles are assembled on such functional surface, the fluorescent light emitted as a result of some reaction will be gathered at the focal point and expected to improve biochemical analysis sensitivity as shown in Fig. 12. The important point is that the mirror finish is not necessary. Instead, regular texture is necessary on it. Using 5-axis milling machine, regular texture can be machined independent of the profile curvature or local inclination of the surface and the finished surface can be applied to the assembly without the need of mirror polishing.

Two parabolic samples, one with texture and another without texture, were produced to examine the effect of texture. The profile of parabolic sample is $20 \mathrm{~mm}$ in diameter and $5 \mathrm{~mm}$ in depth respectively. One sample was cut with a ball end mill $(\phi 10$ $\mathrm{mm}$ ) not to produce texture. Another sample was cut with a square end mill $(\phi 4 \mathrm{~mm})$ to produce texture. Based on the former results, the texturng conditions is determined as pitch 0.1 $\mathrm{mm}$ and slant angle $30^{\circ}$. The tool path was determined as concentric circlular and the corresponding NC code was generated with a CAM software described above.

The profiles were transfered to PDMS casting two times to obtain concave parabolic profile. A fluorine resin was coated on the die before casting so that the PDMS replica can be released easilily from the die. Finaly, the surface was treated with $\mathrm{O}_{2}$ plasma to change the wettability from hydrophobic to hydrophilic just same with the planar sample described above.

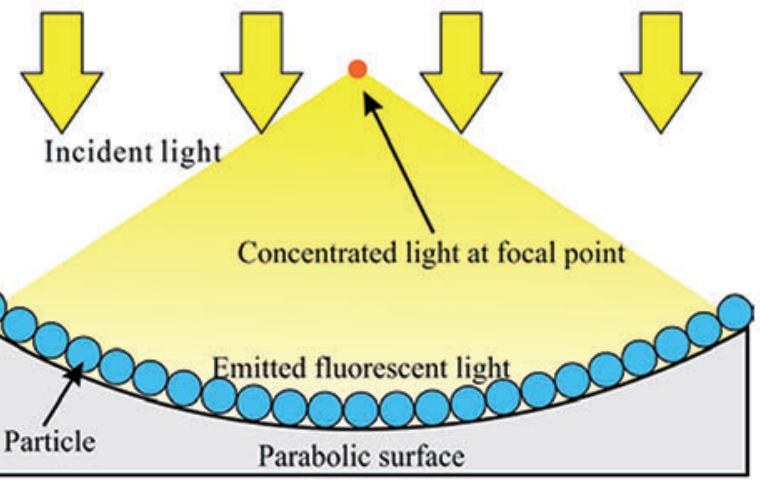

Fig. 12 Concept of light concentration with particles on parabolic surface.

\subsection{Self-assembly on parabolic samples}

Self-assembly conditions was chosen as follows based on the previous results: drawing up speed $12.5 \mathrm{~mm} / \mathrm{s}$, four-times iteration

Figure 13 shows the SEM photos of the self-assembly results on a parabolic surface without texture. Three photos shows the difference in the assemlbies on three different locations. It is suggested that suspension could not be held uniformly on the smooth surface and thus the assembly has become scattered or island like at location (b) and (c) though the assemly has monolayered structure as shown in the magnified view in the inserted figure. At the location (a), the surface looks smooth but the structure is considered as multilayered.

Figure 14 shows the SEM photos of the self-assembly results on a parabolic surface with texture. The aasembly is obviously different from the reults without texture shown in Fig. 13. The assembly is uniform and smooth structure all over the surface independent of the location. In the figure the vertical stripes show the ridges of the texture and particles assemble smoothly around them. Thus it is found that the particle assembly aligned along the concentric texture patterm. It is not easy identify the number of assembly layers, but it is confirmed that the top layer has uniform and packed structure.

Another important result is derived from Fig. 14. On a smooth surface, self-assembly process is strongly affected by the drawing up direction. If so, uniform self-assembly on three-dimensional surface is difficult to obtain. On the other hand, the effect of drawing up direction has weak on the the self-assembly on textured surface. This suggests that texture is effective for asssembly on free-form three-dimensional profiles. 
Parabolic without texture Ball end mill. $p=100 \mu \mathrm{m}$
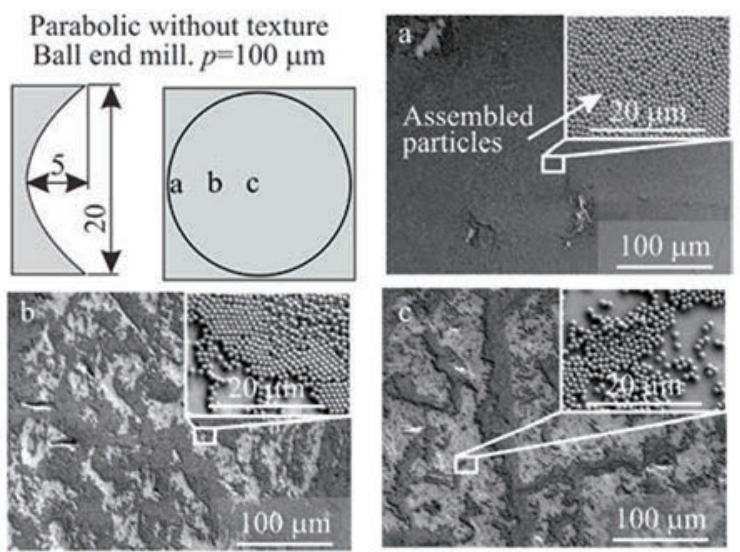

Fig. 13 Self-assembly on parabolic surface without texture.
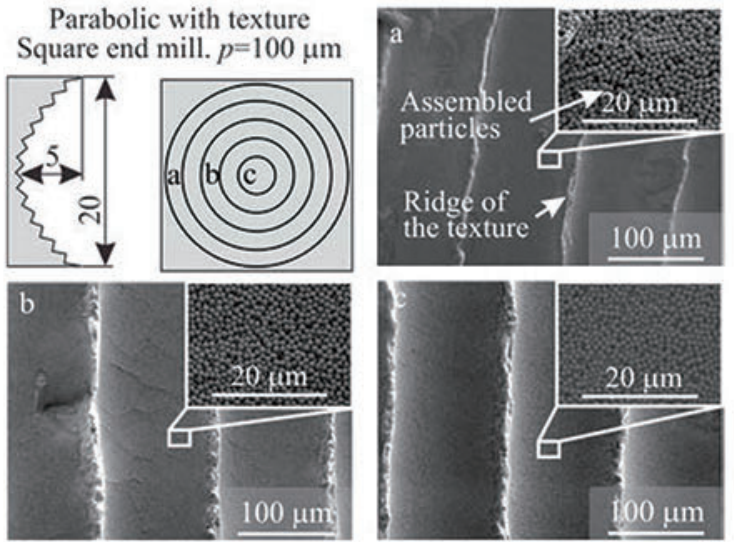

Fig. 14 Self-assembly on parabolic surface with texture.

\subsection{Optical properties}

Figure 15 shows the optical properties of both the planer and the parabolic surface on which particles were assembled. White light was collimated and entered from the backside of the transparent sample through an aperture of $\phi 18 \mathrm{~mm}$, and the light intensity at the distance $d$ apart from the sample on the optical axis was measured with a photomultiplier as shown in the inserted figure. The horizontal axis denotes the distance $d$ and vertical axis denotes the voltage corresponding to the light intensity. The setup is not same with that in Fig. 12, thus the detected light is not fluorescent but the white light scattered at the sample surface. However, optical concentration function can be examined. Each particle on the sample scatters and thus the intensity decays with the increase in the distance $d$ in the case of planner substrate. However, the particles assembled on a parabolic profile can focus the light ideally at the focal point because the scattered light is emitted from the parabolic surface just like the reflected light on a parabolic mirror. It was found that the light was actually concentrated is the case of parabolic surface because the intensity reach its maximum value in the vicinity of focal point, while the intensity of planer substrate is weak and decay monotonously.

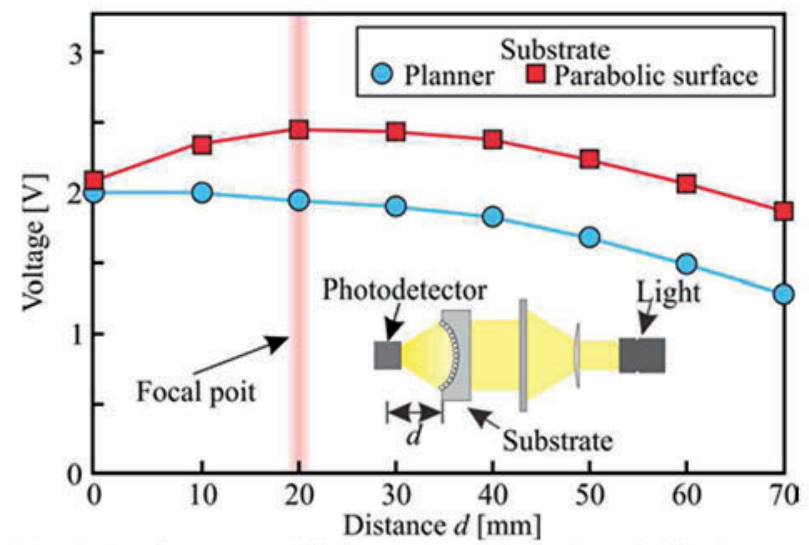

Fig. 15 Performance of light concentration of parabolic element.

\section{Conclusion}

This paper introduced the modification of wettability with texture produced by tool-mark in finishing process and its application to assembly on parabolic surface. The results are summarized as follows:

- Small slant angle and finer pitch is effective to increase drag up and hold the suspension that contains particles and thus to increase coverage of particles

- Texturing on parabolic surface was demonstrated utilizing CAM software and the effectiveness on the assembly was verified

Burr on the texture profile causes unintentional pinning and causes disturbance of the assembly though it is not described in the paper. Measures for this problem are one of the future works.

This work was supported by JSPS KAKENHI Grant Number 22656039.

\section{References}

1) S. Santra et al.: Conjugation of biomolecules with luminophore-doped silica nanoparticles for photostable biomarkers, Analytical chemistry, 73, 20, (2001) 4988.

2) P. Glynne-Jones et al.: Multi-modal particle manipulator to enhance bead-based bioassays, Ultrasonics, 50, 2, (2010) 235 .

3) J.-J. Kim et al.: Biologically inspired LED lens from cuticular nanostructures of frefly lantern, Proceedings of the national academy of sciences, 109, 46, (2012)18674.

4) C. Gang et al.: Refluxing synthesis of anatase $\mathrm{TiO} 2$ nanoparticles assembled microprisms and its application for dye-sensitized solar cells, Science of advanced materials, $\mathbf{6}$, 3, (2014) 459 .

5) X. L. Cheng et al.: $\mathrm{ZnO}$ nanoparticulate thin film: Preparation, characterization and gas-sensing property, Sensors and actuators B, 102, 2, (2004) 248.

6) S. Arnold et al.: Whispering gallery mode carousel - a photonic mechanism for enhanced nanoparticle detection in biosensing, Optics express, 17, 8, (2009) 6230.

7) F. Enrichi et al.: Synthesis and characterization of monodisperse Eu-doped luminescent silica nanospheres for biological applications, Progress in biomedical optics and imaging, 6991, (2008) 69912E.

8) N. D. Denkov et al.: Two-dimensional crystallization, Nature, 361, 6407, (1993) 26.

9) S. Antony et al.: Continuous convective assembling of fine particles into morpho-colored two-dimensional arrays, Langmuir, 12, 5, (1996) 1303.

10) U. Pettersson et al.: Friction and wear properties of micro textured DLC coated surfaces in boundary lubricated sliding, Tribology letters, 17, 3, (2004) 553.

11) S. Mezghani et al.: The effect of groove texture patterns on piston-ring pack friction, Precision engineering, 36, 2, (2012) 210 .

12) N. Moronuki et al.: Frictional properties of the micro-textured surface of anisotropically etched sillicon, CIRP Annals, 52, 1, (2003) 471.

13) F. Llopis et al.: Texture profile and aspect ratio influence on the front reflectance of solar cells, Journal of applied physics, 100, 12, (2006) 124504.

14) N. Moronuki et al.: Design of surface texture for the control of wettability, Transactions of the JSME, 70, 693, (2004) 1244.

15) H. Zhao et al.: Fabrication, surface properties, and origin of superoleophobicity for a model textured surface, Langmuir, 27, 10, (2011) 5927.

16) L. Landau et al.: Dragging of a liquid by a moving plate, Acta Physicochim. URSS, 17, (1942) 42.

17) P. A. Kralchevsky et al.: Capillary forces and structuring in layers of colloid particles, Current opinion in colloid \& interface Science, 6, 4, (2001) 383-401.

18) M. Le Berre et al.: From convective assembly to landau levich deposition of multilayered phospholipid films of controlled thickness, Langmuir, 25, 5, (2009) 2554. 